\title{
Role of Platelet-Rich Plasma injection during Modified Radical Mastectomy
}

\author{
Mahmoud Ibrahim, Abdel Fattah El Sheik, Abdelgaber Rabea \\ Department of General Surgery, Faculty of medicine, Al-Azhar University, Cairo, Egypt
}

*Corresponding author: Abdelgaber Rabea, E-mail: mostafa2712015@ yahoo.com, Mobile No. : 01013223434

Abstract

Background: platelet-rich plasma (PRP) is a human plasma product enriched by platelets, growth factors, and fibrinogen with high hemostatic and healing properties.

Objectives: The aim of this study was to evaluate the effect of autologous PRP on wound healing in women undergoing modified radical mastectomy (MRM).

Patients and Methods: In this randomized and controlled trial, 100 females were admitted to El Sayed Galal hospital, Egypt from October of 2017 to August of 2018 for MRM. The patients were randomly assigned into two groups. The intervention group received PRP after surgery, whereas the control group received the usual care. All patients were evaluated at baseline, five days, and 2 weeks after surgery. The primary endpoint used the REEDA scale for assessing the changes in wound healing. The secondary outcome measures used were the Vancouver scar scale (VSS). All scale scores were analyzed using a repeated measures test for variance.

Results: At the end of study, the PRP group showed a greater reduction in the edema ecchymosed discharge approximation (REEDA) score compared to the control group $(85.5 \%$ reduction in the PRP group; $72 \%$ in the control group) $(\mathrm{P}<0.001)$. Furthermore, patients treated with PRP experienced a 93\% reduction in the VAS score at the end of follow-up, but the control group only observed a $79 \%$ reduction $(\mathrm{P}<0.001)$.

Conclusions: It seems that applying PRP is an effective therapeutic approach for wound healing, and faster wound healing is expected due to the presence of more platelets and growth factors.

Keywords: Platelet-Rich Plasma, Modified Radical Mastectomy, Wound Healing.

\section{Introduction}

Breast cancer is the second most common cause of cancer-related death in women. In most patients, death resulting from either treatment, complications of treatment, or tumor recurrence or metastasis ${ }^{1}$.

The modern approach to breast cancer management is multi-disciplinary. The different surgical treatment options include simple mastectomy, modified radical mastectomy and breast conservative surgery ${ }^{2}$.

Radical mastectomy was first carried out in 1882 and described in 1894 by William Stewart Halsted and for a number of decades it remained the standard operation for early breast cancer. Modified radical mastectomy combines the removal of all breast tissue from the affected breast with lymph node removal from the armpit on the affected side of the body ${ }^{3}$.

Surgical site complications such as infection, hematoma, seroma, dehiscence, and pain may occur. These complications are associated with substantial morbidity and mortality, prolonged hospital stay, and increased cost. Therefore, reduction in the incidence of these morbidities would cut down on medical expenses ${ }^{4}$.

Surgical wounds heal through an orderly sequence of several distinct physiological and biological events that include hemostasis, inflammation, proliferation, epithelialization, fibroplasia, and maturation ${ }^{5,6}$. This process is initiated immediately upon injury. A platelet plaque develops which is composed of platelets and fibrin, and the platelets release granules containing multiple growth factors and thromboxane A2, the latter of which acts as a potent vasoconstrictor. Transforming growth factor beta (TGF-b) is the key growth factor that plays a central role in wound healing. In a wound site, following the clot formation, platelets and mesenchymal stem cells release their complex contents in the wound healing process. The maximum tensile strength of the tissue is reached approximately eight weeks after injury ${ }^{5}$. 
In vitro studies on the proliferation of mesenchymal stem cells (MSC) confirmed that PRP improves MSC proliferation and differentiation, suggesting a high regenerative potential of PRP ${ }^{7}$.

Platelet-rich plasma (PRP) has been used for more than a decade in injectable or gel form ${ }^{8}$, and many studies have demonstrated that PRP stimulates regeneration of the soft tissues (fat, skin, and mucosa) ${ }^{9,10}$ as well as the hard tissues (tendons and bones) ${ }^{\mathbf{1 1}, \mathbf{1 2}}$. However, there are many clinical studies that have been published with a wide range of results and the evaluation of the influence of PRP on wound healing and pain remains variable. Although most studies have concluded that PRP is safe with positive effects, others have indicated conflicting results ${ }^{\mathbf{1 3}}$.

\section{Objectives:}

In this study we attempted to evaluate the efficacy of topical application of autologous PRP in treatment for the wound healing process in patients who underwent modified radical mastectomy.

\section{Patients and Methods}

A study was carried at Al-Azhar university hospitals and Minia insurance hospital on 100 female patients had modified radical mastectomy during the study period from October 2017 to August 2018. Randomly divided into two groups:

Group A:

Classic MRM with PRP injection.

\section{Group B:}

Classic MRM without PRP injection.

Informed consent was taken. Patients were hospitalized the day before surgery and detailed patient history was taken.

Preoperative fitness routine investigations

as $\mathrm{CBC}$, prothrombin time and concentration and ECG were done.

\section{Ethical issues}

Informed consents were taken from all of the patients included in our study. The study was approved by the Ethics Board of Al-Azhar University. Inclusion criteria:

1. Patients more than 15 years of age.

2. Only females presenting to outpatient department.
3. Histopathologically proven cases of infiltrating ductal carcinoma (by presence of malignant cells).

4. Patients with stage I and II of breast cancer.

5. Patients undergoing radical mastectomy.

6. Patients that can be followed up for 2 months.

\section{Exclusion criteria:}

1. Patients with inflammatory breast cancer.

2. Immunocompromised patients such as DM, TB, HIV, other malignant, cardiovascular disorder and patients with a bleeding disorder cases.

3. Patients who received preoperative chemotherapy.

\section{Preparation of Platelet Rich Plasma (PRP):}

By using Buffy coat method

1- Under aseptic conditions, 20 to $30 \mathrm{ml}$ of peripheral blood was drawn in syringes containing anticoagulant (Acid Citrate Dextrose - ACD-A) in the ratio of 3:17 (anticoagulant: whole blood) from patient's anti-cubital vein.

2- Centrifuge WB at a slow speed 1500 for 15 minutes, forming three layers because of its density. The bottom consisting of RBCs, the middle consisting of Platelets, the top PPP (platelets poor plasma).

3- Removing the top layer (PPP) and (upper $2 / 3$ of the upper layer) and transfer the Buffy coat layer (the lower $1 / 3$ \& WBCs layer) into another sterile tube.

4- Centrifuge at high speed 3000 for 10 minutes.

5- Separation of the upper layer (PRP) in sterile syringes.

\section{Treatment procedure:}

- All MRM operations were carried out in a training hospital and under the supervision of senior residents, and all of the patients received preoperative intravenous cephalosporin $(2 \mathrm{~g})$.

- In the operating room before the start of each procedure, PRP was prepared as prescribed above; $4-5 \mathrm{ml}$ of PRP was obtained. 
- All of the procedures were conducted in an operation room with the purpose of safe guarding sterilization.

- Prior to skin closure, PRP was directly applied to the subcutaneous tissue of the wound site by using a sterile syringe. In the control group (group B), the patients received no topical treatment and the subcutaneous tissue was cleaned with normal saline before skin closure.

- After skin closure, a wound dressing with a compressed bandage was applied.

- The patients were examined by the physicians on day 2 , and then five days and eight weeks after the procedure.

- The wound healing was evaluated by using the Vancouver scar scale (VSS) and the edema ecchymosed discharge approximation (REEDA) scale ${ }^{(\mathbf{P D}, \mathbf{1 9 9 0})}$.

\section{Outcomes}

The primary endpoint used the REEDA scale for assessing the changes in wound healing. REEDA as a descriptive scale has 4 points in a categorical score that measures 5 items of healing:

Redness (hyperemia), edema, ecchymosis, discharge, and approximation of the wound edges (cooptation).

Each item is rated on a scale of 0 to 3 , and total scores may range from 0 to 15 . A lower score indicates better healing ${ }^{14}$.

The secondary outcomes were measured by Vancouver Scar Scale (VSS) which used to detect formation of keloids or hypertrophic scars.

It assesses 4 subjective variables:

Vascularity, height/thickness, pliability, and pigmentation within a possible range of 0 - 14 for the total score ${ }^{15}$.

Statistical analysis was performed by using computer software SPSS 18 for windows. The endpoint mean of the REEDA and VSS were analyzed using repeated measures analysis of variance Values of $\mathrm{P}=0.05$ were considered statistically significant.

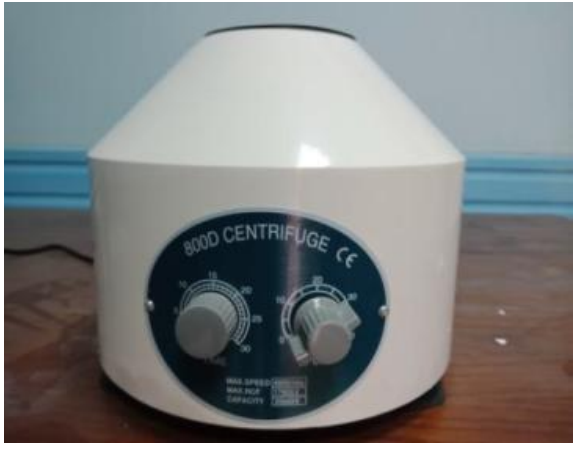

Fig (1) Centrifuge device.

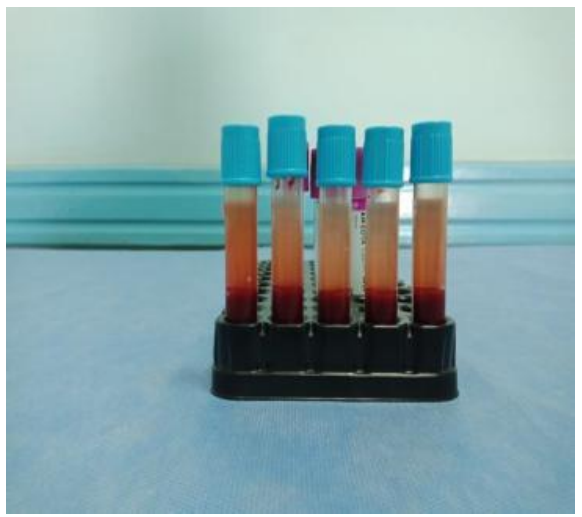

Fig (2) Sample of Whole blood.

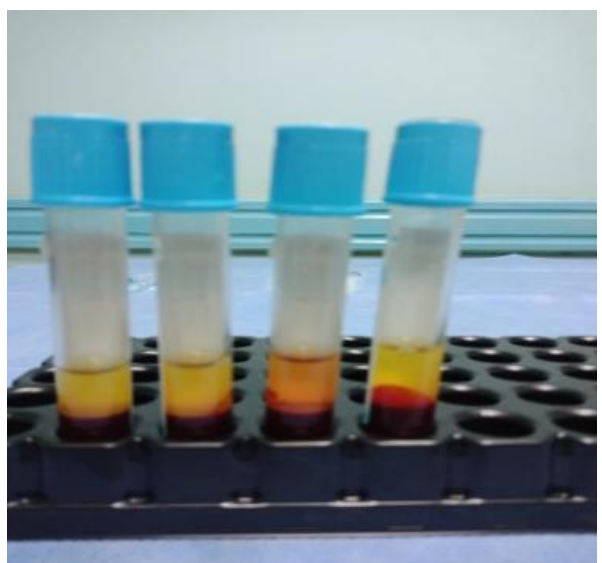

Fig (3) after separation of whole blood on 3000 speed forming 3 layers.

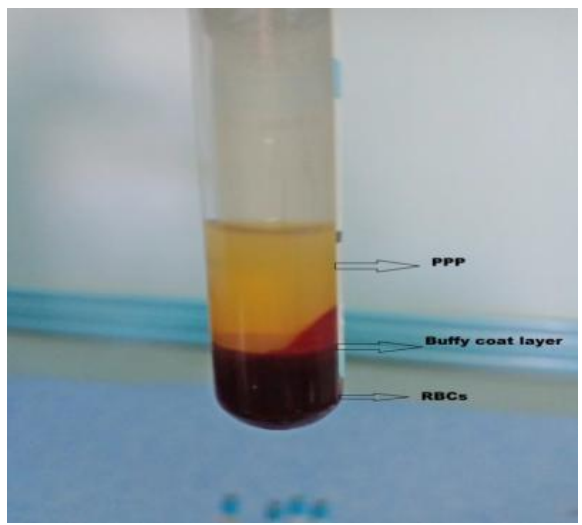

Fig (4) 3layer after 1st centrifuge. 


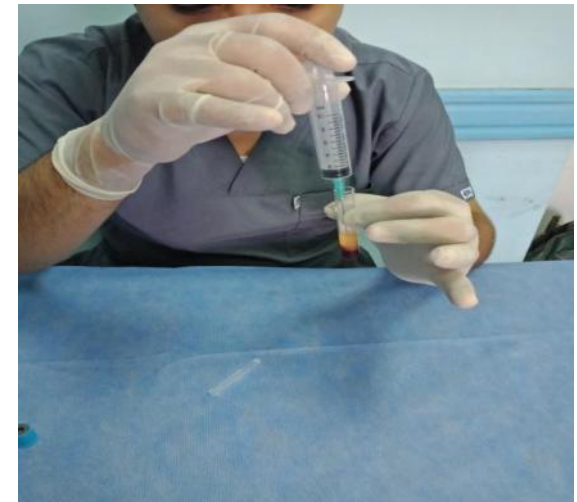

Fig (5) separation of buffy coat Layer.

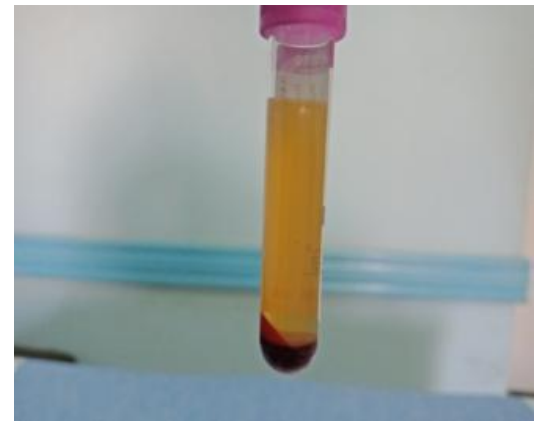

Fig (6) After $2^{\text {nd }}$ centrifuge forming PRP.

\section{Result}

A total of 100 female patients had modified radical mastectomy.

\section{Group (A):}

32 prospective cases had modified radical mastectomy with PRP injection. Average age 52.25 years.

Table 1: Age group in Group A

\begin{tabular}{|l|l|}
\hline Age group & No. of cases \\
\hline $21-30$ & 1 \\
\hline $31-40$ & 6 \\
\hline $41-50$ & 7 \\
\hline $51-60$ & 12 \\
\hline $61-70$ & 6 \\
\hline
\end{tabular}

18 cases had Right MRM.

14 cases had Left MRM.

Group (B):

68 cases had modified radical mastectomy without PRP injection.

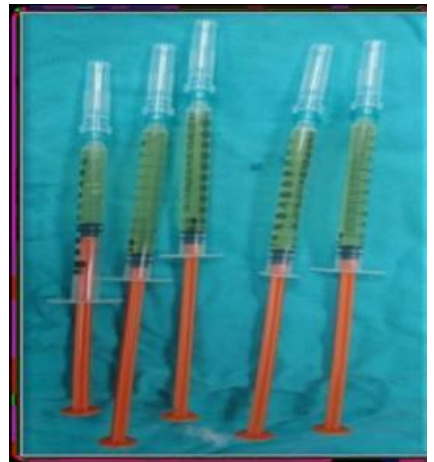

Fig (7) Separation of PRP in syringes ready for injection.

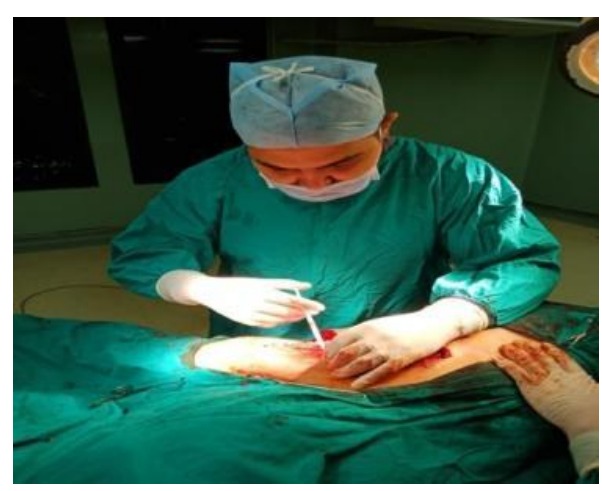

Fig (8) PRP injection in the edges of the wound.

Twenty one cases prospective \& 47 cases retrospective. Average age was 51.32 years.

Table 2: Age group in Group B

\begin{tabular}{|l|l|}
\hline Age group & No. of cases \\
\hline $21-30$ & 1 ( retrospective) \\
\hline $31-40$ & $5($ retrospective $)$ \\
\hline $41-50$ & $21(5$ pro. $\& 16$ retro. $)$ \\
\hline $51-60$ & $32(9$ pro. $\& 23$ retro. $)$ \\
\hline $61-70$ & $9(7$ pro. \& 2 retro. $)$ \\
\hline
\end{tabular}

42 cases had Right MRM.

26 cases had Left MRM.

The means of the primary and secondary outcomes for three measured times for both groups are shown in (Table 3). In the analysis of the overtime scores, the PRP group had lower scores compared to control group for all measures.

After five days, the reduction in the REEDA score for the PRP group was 1.03, a $43 \%$ reduction. This trend was constant overtime and the results after eight weeks showed a decrease of 0.57 , a $42.5 \%$ 


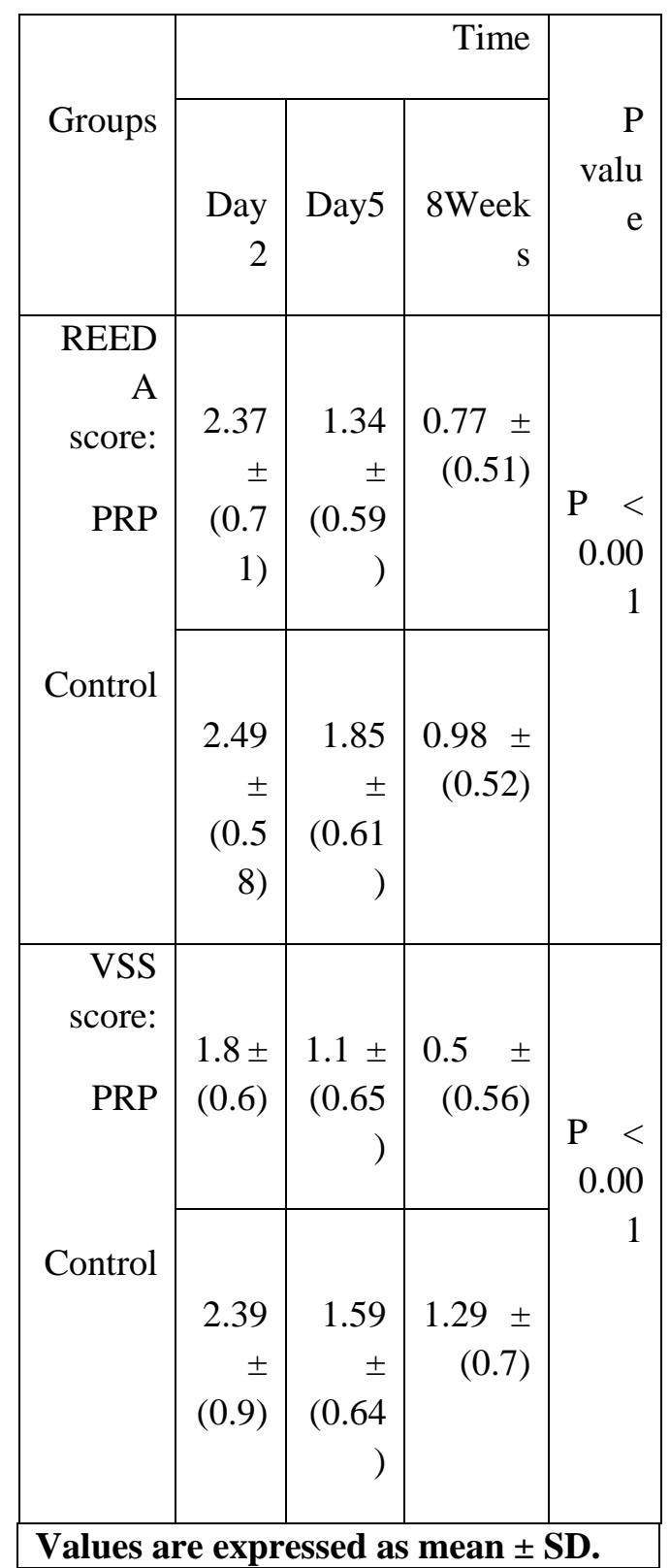

reduction. A similar trend was observed in the control group: after five days, the

reduction in the REEDA score was 0.64, a $25 \%$ reduction, and after eight weeks, there was a 0.87 decrease, or a $47 \%$ reduction. Fig (9).

The general analysis with repeated measures showed that treatment with PRP had a significant effect on reducing the REEDA score compared with the treatment of the control group $(\mathrm{F}(1,132)=7.28, \mathrm{P}=$ 0.008).

Patients treated with PRP experienced a 1.7 decrease, or a $42 \%$ reduction, in the VSS score at 5 days, and after 8 weeks of the study, the observed decline was 1.17 , a
$51 \%$ reduction. A similar trend was observed in the control group: after 5 days, there was a decrease of 1.53 , a $31 \%$ reduction, and after eight weeks, there was a 1.6 decrease, or a $48 \%$ reduction. Fig (10).

Repeated measures analysis showed a significant effect on the VSS score in favor of PRP $(F(1,132)=80.15, \mathrm{P}<0.001)$.

Table 3: Means of REEDA and VSS Scores for the Two Groups during the Study.

Fig (9) Improvement from the Baseline in the Means of the REEDA Scores over Time.

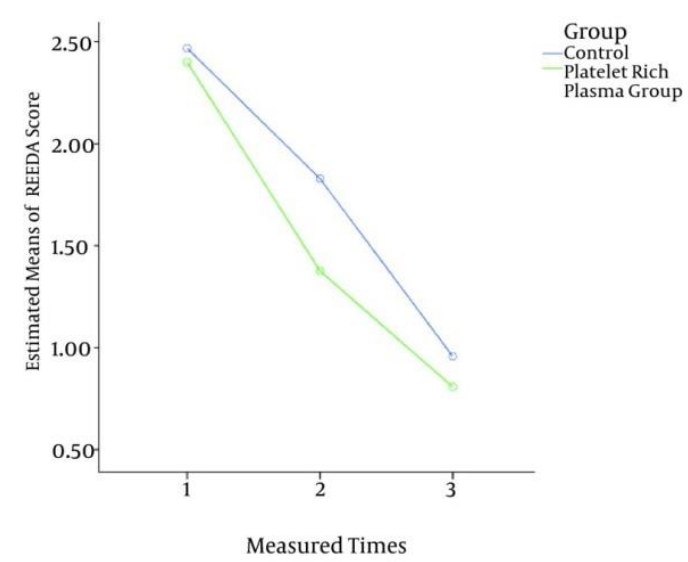

Fig (10) Improvement from the Baseline in the Means of the VSS Scores over Time.

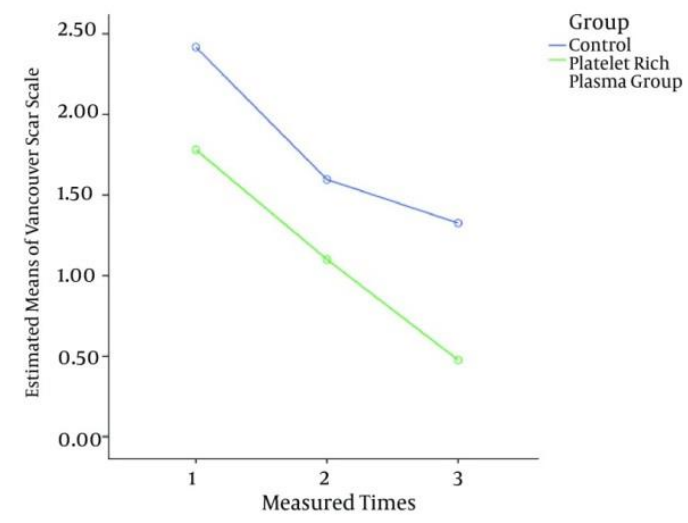


Table 4: percentage of complications occurred in PRP group

\begin{tabular}{|l|l|l|}
\hline Complications & $\begin{array}{l}\text { No. of } \\
\text { cases }\end{array}$ & \\
\hline Seroma & 7 & 21.88 \\
\hline Infection flap & 2 & 6.25 \\
\hline $\begin{array}{l}\text { Skin } \\
\text { necrosis }\end{array}$ & - \\
\hline Paresthesia & 3 & 9.375 \\
\hline Dehiscence & 1 & 3.1 \\
\hline
\end{tabular}

Table 5: percentage of complications occurred in control group

\begin{tabular}{|l|l|l|}
\hline Complications & $\begin{array}{l}\text { No. of } \\
\text { cases }\end{array}$ & $\%$ \\
\hline Seroma & 21 & 30.88 \\
\hline Infection & 5 & 7.35 \\
\hline Skin flap necrosis & 2 & 2.94 \\
\hline Paresthesia & 11 & 16.18 \\
\hline Dehiscence & 1 & 1.47 \\
\hline
\end{tabular}

Suction drains was removed in PRP group (average $9 \pm 2$ days), but in control group (average $11 \pm 2$ ).

No. of patients suffered from chronic seroma that needed further post-operative aspiration in PRP group 2 cases $(6.25 \%)$.

No. of patients suffered from chronic seroma that needed further post-operative aspiration in control group 25 cases (36.76 $\%)$.

\section{Discussion}

Mainstay of breast cancer management is surgery directly or after neoadjuvant chemotherapy. The surgical treatment for the breast cancers depends upon the stage of disease at the time of initial presentation, age of patients, patient's preference and surgeon's choice. Among the procedures, modified radical mastectomy with axillary clearance is the most commonly performed surgery. Although it is commonly performed procedure in any surgical setup still it is associated with significant complications that increase morbidity and mortality rate.

Topical application of PRP as a novel method with the potential to prevent postoperative wound infection, enhance the wound healing process, and reduce pain and other adverse events has been acknowledged by the surgical community.

PRP is a volume fraction of blood having a high concentration of platelets above the baseline that markedly improves the adhesive properties and the process of wound healing.

After application of PRP, the tissue-healing substances are released. The supraphysiological concentration of platelets at the wound site accelerates the healing process and protects the wound against the infection.

Because of its physiological role in wound healing, PRP is being used more often for a variety of clinical applications, and it is now popular to apply it as a part of routine treatment. However, it still remains uncertain whether the impact of topical application of PRP is a fact or a fiction.

In our study we found that patients who were treated with topical autologous PRP (group A) had a significant reduction in pain, keloids, and hypertrophic scar formation, and they experienced better wound healing after surgery in comparison with the control group (group B) $(\mathrm{P}<$ 0.0001).

Other studies similar to the one presented here have concluded that PRP improves the wound healing process ${ }^{16,17}$. Everts et al, applied platelet-leukocyte gel on 40 patients who underwent open subacromial surgery and showed that the VAS for pain had been decreased. They also demonstrated a significant reduction in recovery time and analgesic usage during the 6-week follow-up. The VAS score was $2.0_{-} 2.0$ and $1.1_{-} 0.3$ in the control and treatment groups, respectively, which were the same as the present study, whereas the mean VAS in the PRP and the control group at the first day, fifth day, and eighth week after surgery were $0.6 \_0.07,0.96$ 0.11 , and $2.2_{-} 0.79$ vs. 3.3 _ 1.2 , and 1.11 _ 0.84 vs. 1.7 _ 0.75 , respectively. This 
study did not evaluate analgesic usage and dose. (Everts PA, 2002).

In 1986, Knighton et al. ${ }^{20}$ showed that the accelerated epithelialization of granulation tissue leading to complete repair of chronic nonhealing ulcers is attainable by the use of autologous platelet factors. This was the first clinical demonstration that locally acting factors derived from autologous blood promote healing of chronic cutaneous ulcers. In this study, the time to $100 \%$ healing after initiation of plateletderived wound-healing factors (PDWHF) was $7.5 \pm 6.5$ weeks.

There was a direct correlation between the initiation of PDWHF therapy and 100\% healing. The age of the patients and the location of the ulcers had no statistically significant effect on PDWHF-stimulated wound repair.

A study conducted by Frykberg et al. ${ }^{23}$ on 49 patients with 65 nonhealing ulcers showed that 63 of 65 ulcers responded with a reduction in area, volume and undermining of the ulcers in a mean duration of 2.8 weeks with 3.2 treatments.

Steenvoorde et al. ${ }^{24}$ conducted a study on 12 patients with 13 wounds, showing that 7 of 13 wounds required more than 1 application, with a mean number of 2.2 applications and a mean treatment period of 4.2 weeks.

Kakudo et al. ${ }^{25}$ treated five cases of intractable skin ulcer with autologous PRP, among which three ulcers healed completely within 4 weeks and epithelization of wound occurred within 6.6 weeks on average.

However, in contrast to the results of this study, some authors have shown the failure of PRP in promoting wound healing. One such study was a double blind randomized and controlled trial which used autologus platelet gel after total knee arthroplasty on 102 patients with a 3-month follow up. The authors have concluded that there was no positive effect of the autologus platelet gel on wound healing. They have also indicated that it had no effect on pain or hemoglobin values ${ }^{12,13,19 .}$

According to Marx, to truly concentrate platelets from autologous blood, the device must use a double centrifugation technique 21. Regardless of the rate of centrifugation or the time of centrifugation, a single spin cannot adequately concentrate platelets, because the red blood cells will interfere with their fine separation ${ }^{22}$.

\section{The present study had the following limitations:}

First, it was not double-blind, and this could have generated biases.

Second, it had a short-term follow-up period.

Third, we did not investigate the effects of varying platelet concentrations.

Currently, there are numerous studies with variable evidence. Variants can be related to many factors including health status, donor variability, centrifuge duration and speed, platelet concentration, or different types of wounds and tissues, among other factors.

Clearly it is necessary to perform further multicentric, controlled, and double-blind clinical trials with similar and standard protocols to assess the potential influences of PRP until more conclusive evidence.

\section{Conclusion}

The present study is the first prospective, randomized, and controlled trial evaluating the efficacy of autologous PRP in breast surgery, and it has demonstrated that PRP has positive effects on wound healing and infection reduction in patients undergoing MRM.

Clearly it is necessary to perform further multicentric, controlled, and double-blind clinical trials with similar and standard protocols to assess the potential influences of PRP until more conclusive evidence

\section{References}

1. Society A C(2008): Cancer facts \& figures. The Society, 26: 2198-2204

2. Franceschini G, A M Sanchez, A Di Leone, S Magno, F Moschella, C Accetta, R Masetti(2015): New trends in breast cancer surgery: a therapeutic approach increasingly efficacy and respectful of the patient. Il Giornale di chirurgia, 36: 145.

3. Halsted $\mathbf{C} \mathbf{P}, \mathbf{J} \mathbf{R}$ Benson, I Jatoi(2014): A historical account of breast cancer surgery: beware of local recurrence but be not radical. Future Oncology, 10: 1649-1657. 
4. Fanning J, Murrain L, Flora R, Hutchings T, Johnson JM, Fenton BW(2007): Phase I/II prospective trial of autologous platelet tissue graft in gynecologic surgery. J Minim Invasive Gynecol., 14: 633-7.

5. Jones HW, Rock JA, TeLinde RW(2015): Te Linde's operative gynecology. Lippincott Williams and Wilkins.

6. Diegelmann RF, Evans MC(2004): Wound healing: an overview of acute, fibrotic and delayed healing. Front Biosci., 9:283-9.

7. Mishra A, Tummala $P$, King A, Lee B, Kraus M, Tse $\mathrm{V}$ et al.(2009): Buffered platelet-rich plasma enhances mesenchymal stem cell proliferation and chondrogenic differentiation. Tissue Eng Part C Methods, 1:431-5.

8. Man D, Plosker H, Winland-Brown JE(2001): The use of autologous platelet rich plasma (platelet gel) and autologous platelet-poor plasma (fibrin glue) in cosmetic surgery. Plast Reconstr Surg., 107: 229-37.

9. Balbo R, Avonto I, Marenchino D, Maddalena L, Menardi G, Peano G(2010): Platelet gel for the treatment of traumatic loss of finger substance. Blood Transfus., 8: 255-9.

10. Chen TM, Tsai JC, Burnouf T(2010): A novel technique combining platelet gel, skin graft, and fibrin glue for healing recalcitrant lower extremity ulcers. Dermatol Sur., 36: 453-60.

11. Nacopoulos C, Dontas I, Lelovas P, Galanos A, Vesalas AM, Raptou P et al.(2014): Enhancement of bone regeneration with the combination of platelet-rich fibrin and synthetic graft. J Craniofac Surg., 25: 2164-8.

12. Castricini R, Longo UG, De Benedetto M, Panfoli N, Pirani P, Zini $R$ et al.(2011): Platelet-rich plasma augmentation for arthroscopic rotator cuff repair: a randomized controlled trial. Am J Sports Med., 39: 258-65.

13. Silva A, Sampaio R(2009): Anatomic ACL reconstruction: does the platelet rich plasma accelerate tendon healing?. Knee Surg Sports Traumatol Arthrosc., 17: 676-82.
14. Hill PD(1990): Psychometric properties of the REEDA. $J$ Nurse Midwifery, 35: 162-5.

15. Nedelec B, Shankowsky HA, Tredget EE(2000): Rating the resolving hypertrophic scar: comparison of the Vancouver Scar Scale and scar volume. J Burn Care Rehabil., 21: 205-12.

16. Serra R,BuffoneG, Dominijanni A, Molinari V,MontemurroR, de Franciscis S(2013): Application of platelet-rich gel to enhance healing of transmetatarsal amputations in diabetic dysvascular patients. IntWound J., 10: 612-5.

17. Gomez-Caro A, Ausin P, Boada M(2011): Platelet rich plasma improves the healing process after airway anastomosis. Interact Cardiovasc Thorac Surg., 13: 552-6.

18. Everts PA, Devilee RJ, Brown Mahoney C, van Erp A, Oosterbos CJ, Stellenboom M, et al(2008): Exogenous application of platelet leukocyte gel during open subacromial decompression contributes to improved patient outcome. A prospective randomized double blind study. Eur Surg Res., 40: 203-10.

19. Peerbooms JC, de Wolf GS, Colaris JW, Bruijn DJ, Verhaar JA(2009): No positive effect of autologous platelet gel after total knee Review of literature 116 arthroplasty. Acta Orthop., 80: 557-62.

20. Knighton DR, Ciresi KF, Fiegel VD, Austin LL, Butler EL(1986): Classification and treatment of chronic nonhealing wounds. Successful treatment with autologous plateletderived wound healing factors (PDWHF). Ann Surg., 204: 322-30.

21. Marx RE(2001): Platelet-rich plasma (PRP): what is PRP and what is not PRP?. Implant Dent., 10: 225-8.

22. Bhanot S, Alex JC(2002): Current applications of platelet gels in facial plastic surgery. Facial Plast Surg., 18: 27-33.

23. Frykberg RG, Driver VR, Carman D, Lucero B, Borris-Hale C, Fylling CP, Rappl LM, Clausen PA(2010): Chronic wounds treated with a physiologically relevant concentration of platelet-rich plasma gel: a 
prospective case series. Ostomy Wound Manage, 56: 36-44.

24. Steenvoorde $P$, van Doorn LP, Naves C, Oskam J(2008): Use of autologous plateletrich fibrin on hard-to-heal wounds. J Wound Care, 17: 60-3.
25. Kakudo N, Kushida S, Ogura N, Hara T, Suzuki K(2012): The use of autologous platelet rich plasma in the treatment of intractable skin ulcer. Open J Reg Med., 1: 29-32 